www.cya.unam.mx/index.php/cya

Contaduría y Administración, 64 (4), Especial Gobierno Corporativo, 2019, 1-17

\title{
Determinantes de la divulgación de información en las universidades latinoamericanas para un buen gobierno corporativo
}

\author{
Determinants of information disclosure in latin american \\ universities for good corporate governance \\ Juan Bautista Abello-Romero $\otimes^{*}{ }^{*}$, Claudio Mancilla ${ }^{1}$, \\ Francisco Ganga-Contreras ${ }^{1}$, Juan Guillermo Estay-Sepúlveda ${ }^{2}$ \\ ${ }^{1}$ Universidad de Los Lagos, Chile
${ }^{2}$ Universidad Católica de Temuco y Universidad Adventista de Chile, Chile \\ Recibido el 6 de febrero de 2019; aceptado el 10 de marzo de 2020 \\ Disponible en Internet el: 11 de junio de 2020
}

\section{Resumen}

En el entendido de que la divulgación de información conlleva una serie de beneficios para la sociedad, este trabajo responde a la pregunta por las causas de que se divulguen distintos niveles de información por parte de universidades latinoamericanas. En la investigación se propone un modelo teórico y cuatro dimensiones (y variables) para comprender los niveles de divulgación voluntaria de la información en dichas instituciones. Se estudiaron 219 universidades de cinco países latinoamericanos, reconocidas dentro de las 500 mejores instituciones de la región, y se utilizó un modelo de regresión lineal múltiple para testear las distintas hipótesis. Los resultados validan tanto el modelo teórico como sus dimensiones, con implicancias teóricas, empíricas y de política pública.

Código JEL: I23, G30, D82

Palabras clave: Determinantes de la divulgación de información; Universidades latinoamericanas; Gobiernos corporativos de las universidades; Teoría de gobiernos corporativos

\footnotetext{
* Autor para correspondencia

Correo electrónico jabello@ulagos.cl (J.B. Abello Romero).

La revisión por pares es responsabilidad de la Universidad Nacional Autónoma de México.

http://dx.doi.org/10.22201/fca.24488410e.2019.2449

0186- 1042/@ 2019 Universidad Nacional Autónoma de México, Facultad de Contaduría y Administración. Este es un artículo Open Access bajo la licencia CC BY-NC-SA (https://creativecommons.org/licenses/by-nc-sa/4.0/)
} 


\begin{abstract}
In the context that the disclosure of information brings benefits for society, this work answers the questions of what are the causes for different levels of disclosure of information by Latin American universities. The research proposes a theoretical model and four dimensions (and variables) to understand the levels of voluntary dissemination of information. A total of 219 universities from five Latin American countries were considered to answer the research question; these universities are recognized within the 500 best institutions in the Region. Also, a multiple linear regression model was used to test the different hypotheses. The results validate both the theoretical model and its dimensions, which leads to theoretical, empirical and public policy implications.
\end{abstract}

JEL code: 123 , G30, D82

Keywords: Determinants of information disclosure; Latin American universities; corporate governments of universities; Corporate governance theory

\title{
Introducción
}

La educación superior en América Latina ha estado en constante deliberación respecto de la calidad de las universidades, expresada en la formación que entregan y el conocimiento que generan. La Organización de Estados Iberoamericanos para la Educación, la Ciencia y la Cultura (OEI, 2010), en su definición de las metas educativas para el 2021, indica que el desarrollo de las naciones depende ahora más que nunca de la calidad de la formación a la que se accede en las universidades y del conocimiento que se pueda generar y acumular en ellas.

En el anterior contexto, las universidades producen diversos tipos de bienes, como los bienes públicos (Garrity, 2015) y bienes comunes (Frost y Hattke, 2018). Los bienes públicos se caracterizan por no ser competitivos ni excluibles (Marginson, 2017). En cambio, los bienes comunes son recursos colectivos que benefician a todos los miembros de la institución, independientemente de si han contribuido a la creación de ese recurso o no (Frost y Hattke, 2018). Los bienes públicos requieren de la cooperación de los miembros de la sociedad y los bienes comunes universitarios no se pueden producir sin una acción colectiva, lo que reafirma la necesidad de un control social, sobre todo si los bienes son financiados por el gobierno nacional o local y entidades filantrópicas, como sucede con los bienes públicos e incluso parcialmente públicos. En estos casos el control social lo ejerce la sociedad y/o los distintos grupos de interés. En este punto, la divulgación de la información juega un papel fundamental en el control social, pues tiene una baja rivalidad en el consumo (Frost y Hattke, 2018), la que al ser distribuida se enriquece. Sin embargo, esto no implica que existan actores dentro de la universidad que consideren que la acumulación de información les beneficie o que el compartir estos recursos no les convenga.

La información es un factor esencial en la toma de decisiones de la organización y de las distintas partes interesadas. Su divulgación acarrea una serie de beneficios, tales como mejoras en la transparencia organizacional, lo que posibilita mayores niveles de confianza con los diversos grupos de interés. Otro beneficio es la disminución de los niveles de asimetría de información, lo que impacta en la disminución de los costos de agencia (García-Sánchez y NogueraGámez, 2017; Van Buskirk, 2012; Alves et al., 2015). La divulgación de información es una decisión estratégica para las organizaciones (Rodríguez et al., 2010; Molio, 2016), a la vez que puede mejorar la gobernabilidad (Cormier et al., 2010). En conjunto con la transparencia, representa uno de los principios de los gobiernos corporativos (OCDE, 2016).

Frente a ello, surge la siguiente interrogante: ¿cuáles son las causas de que se divulguen distintos niveles de información? Para esto se propone un modelo teórico sustentado por diversas teorías organizacionales y una validación empírica de las variables explicativas. 


\section{J. B. Abello Romero, et al. / Contaduría y Administración, 64(4) Especial Gobierno Corporativo, 2019, 1-17}

\section{http://dx.doi.org/10.22201/fca.24488410e.2019.2449}

Las variables se tipifican en cuatro dimensiones: (1) características propias de la universidad, su trayectoria, su tamaño, tipo de propiedad, etc.; (2) capacidad de generar información, dado que se puede tener la mejor voluntad de divulgarla, se cuente o no con los recursos para hacerlo; (3) partes interesadas apropiativas; y (4) atributos de los Gobiernos Corporativos de las Universidades.

Este trabajo está en línea con las propuestas realizadas en Abello y Mancilla (2018), ampliando su base teórica. En materia empírica, agrega lo aportado por Abello et al. (2018) sobre las variables que reflejan las características propias de la universidad y avanza en una propuesta explicativa que permite ampliar los factores que afectan los niveles de la divulgación de información de las universidades latinoamericanas hacia los distintos grupos de interés.

Para afrontar la pregunta de investigación se realizó un meticuloso proceso de recopilación de antecedentes de 219 universidades de cinco países latinoamericanos que se posicionan dentro de las 500 instituciones mejor ubicadas en el ranking Scimago. Los casos estudiados representan el $83 \%$ de las universidades latinoamericanas, y en materia de publicaciones científicas el $89 \%$ del total de América Latina.

\section{Revisión de la literatura e hipótesis}

En concordancia con lo expresado por Abello y Mancilla (2018), se debe recurrir a un modelo multi-teórico basado en teorías organizacionales como la teoría de agencia, servidores, partes interesadas, institucional, dependencia de los recursos y la de mandos superiores para establecer las determinantes de la divulgación de información en organizaciones complejas como las universidades.

La teoría de agencia (Jensen y Meckling, 1976) sostiene que los directorios de las organizaciones son importantes para monitorear el desempeño, lo que lleva a una mayor divulgación de información (Gianiodis y Markman, 2016; Pérez-Calero et al., 2017; Kultys, 2016; Rodrigues et al., 2016).

La teoría de servidores (Davis et al., 1997) admite que el equipo rectoral posea una motivación intrínseca capaz de maximizar el valor de la institución (Deb y Wiklund, 2017; Madison et al., 2016), en donde los miembros de los directorios se posicionan como colaboradores y ejercen una función de asesoramiento (Pucheta-Martínez et al., 2016), por lo que no es extraña la divulgación voluntaria de información, incluso ante la existencia de dualidad en la estructura del liderazgo (John et al., 2016; Pham et al., 2015).

La teoría de las partes interesadas (Freeman, 1984) sugiere que la empresa es responsable de servir a todos los grupos de interés que se ven afectadas por las decisiones y las actividades de la universidad (Freeman et al., 2004; Andersen, 2015), dando lugar a la presentación de informes (divulgación de la información) dirigidos a grupos más amplios que requieran de una mayor transparencia en pos de ganar la confianza de los grupos influidos, lo que conduce a un mejor rendimiento a largo plazo (Ferrero-Ferrero et al., 2018).

La teoría institucional (Dimaggio y Powell, 1983) aborda la influencia de los mecanismos de gobernanza externos, los que inciden en los mandos superiores de transparencia mediante una mayor divulgación de la información en las universidades, entidades acreditadoras, organismos que establecen políticas públicas sobre educación superior y las partes interesadas (Harris, 2013; Fay y Zavattaro, 2016; Fowles et al., 2016; Nurunnabi, 2015).

La teoría de dependencia de recursos (Pfeffer y Salancik, 1978) da por sentado que una función de los miembros del MCC es generar acceso a recursos externos, ya que ninguna organización es capaz de generar todo lo que necesita. Por lo tanto, se consideran figuras como la de los directores independientes y diversos vinculados a redes que posibilitan el acceso a estos recursos externos, lo que afecta la composición del MCC e incrementa la divulgación.

La teoría de mandos superiores (Hambrick y Mason, 1984) aporta desde la perspectiva del comportamiento de los miembros del MCC, considerando que son personas con racionalidad limitada y sus acciones se ven afectadas por su capital social y humano, por ello una composición diversa del MCC posibilita cumplir con mayor eficiencia los roles de 
asesoramiento y acceso a nuevos recursos (Krause et al., 2016; Wei et al., 2018; Hoskisson et al., 2017; Plöckinger et al., 2016; Ruiz-Jiménez y Fuentes-Fuentes, 2016; Shi et al., 2017; Kuo et al., 2018; Sun et al., 2019).

La figura 1 es el esquema del modelo multi-teórico que sustenta la relación lineal entre las variables que, de acuerdo a esta investigación, explican los niveles de divulgación de información de las universidades latinoamericanas. Estas variables se agrupan en cuatro dimensiones: a) los atributos de los Máximos Cuerpos Colegiados (MCC); b) variables que caracterizan las universidades; c) grupos de interés apropiativos; y d) capacidad de generar información.

Obsérvese en la figura que las distintas dimensiones se han creado a partir de las cinco teorías indicas al inicio de esta sección. A su vez, estas teorías se sustentan en los distintos requerimientos de los grupos interesados (entorno, ambiente o sociedad). La teoría de mandos superiores no tiene un vínculo directo con los grupos interesados, ya que se centra en los miembros de los MCC. Finalmente, estas dimensiones tendrían una incidencia sobre la divulgación de información, que es en lo que se centra este trabajo, y repercutirían en una mayor transparencia y disminución de los niveles de asimetría.

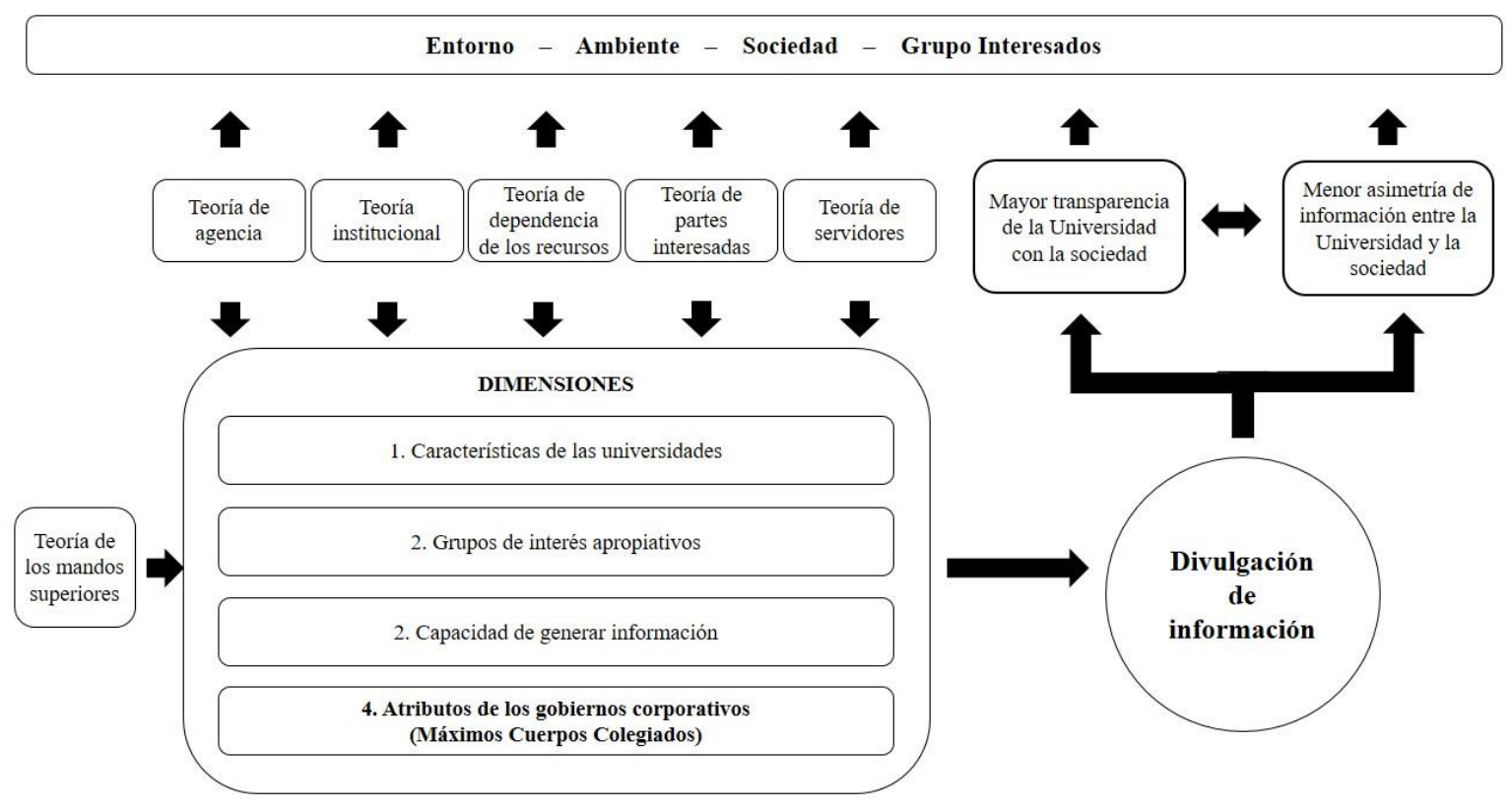

Figura 1. Esquema multi-teórico de la divulgación de información

Fuente: elaboración propia.

\section{Variables que explican la divulgación de información}

Tal como se ha indicado previamente, las variables explicativas que forman parte del modelo se categorizan en cuatro dimensiones: (1) características de las universidades, representada en variables tales como tamaño, antigüedad, capacidad de investigar y la pertenencia de los derechos residuales (propiedad), (2) la dimensión de los grupos de interés apropiativos, como los académicos, estudiantes, funcionarios y egresados, (3) capacidad para generar información por parte de las universidades y (4) atributos de los gobiernos corporativos representados por los directorios de la universidades denominados Máximos Cuerpos Colegiados (MCC), tales como tamaño del MCC, independencia y diversidad que recogen la composición de los MCC, y la estructura de liderazgo de los MCC. 


\section{J. B. Abello Romero, et al. / Contaduría y Administración, 64(4) Especial Gobierno Corporativo, 2019, 1-17}

http://dx.doi.org/10.22201/fca.24488410e.2019.2449

\section{Características de las universidades}

Las universidades latinoamericanas se pueden caracterizar o tipificar a partir de las siguientes variables: la propiedad, tamaño, trayectoria y su capacidad de investigar. Este último aspecto puede ser relevante al momento de caracterizar las universidades latinoamericanas, tal como Abello et al. (2018) explicitan.

La condición estatal de las universidades implica que estén sujetas a escrutinios adicionales, lo que determina un aumento de los costos de agencias. La divulgación de información resulta un buen paliativo para los costos de agencias (Nagata y Nguyen, 2017; Khlif et al., 2017; Sabbaghi, 2016; Alnabsha et al., 2018). La magnitud o tamaño de la organización es una característica que afecta en muchos ámbitos a las organizaciones. En el plano de la divulgación de información, las universidades de mayor tamaño son más visibles para el público, lo que las hace más propensas a la crítica y la atención de los organismos reguladores. A su vez, esta exposición pública obliga a las entidades de mayor tamaño a realizar divulgación voluntaria de sus actividades (Garde-Sánchez et al., 2017; Elshandidy y Shrives, 2016; Felix et al., 2016; Testera y Cabeza, 2013).

La búsqueda de la ciencia prioritariamente tiene lugar en las universidades (Vasbinder, 2017), esto implica generar las capacidades para investigar en estas instituciones. La necesidad de recursos para generar las capacidades de investigación obliga a las universidades, independientemente de su condición, a divulgar información a los diversos stakeholders (Arechavala-Vargas y Sanchez-Cervantes, 2017; Mtawa et al., 2016; Harsh et al., 2018; Kohoutek et al., 2017; Powell y Dusdal, 2017; Huenneke et al., 2017; Kotsemir y Shashnov, 2017; Brownson et al., 2017; Rhoads et al., 2017).

La trayectoria como característica de las universidades se puede justificar por la permanencia en el sistema, y en la medida en que pasa el tiempo, consigue explicitar mejor su quehacer misional y su desempeño. La antigüedad en el negocio se ha considerado como una adecuada variable en estudios sobre la divulgación y sus determinantes (Dolinsek y Lutar-Skerbinjek, 2018; Garas y Elmassah, 2018; Yasser et al., 2017). La cantidad y calidad de la información divulgada por una universidad ayuda a incrementar su credibilidad, reputación y consolidar su trayectoria. El incremento de la trayectoria y la reputación generan mayores posibilidades de establecimiento de redes y conexiones con instituciones similares a nivel nacional o internacional, lo que a su vez implica un mayor acceso a nuevos recursos y la necesidad de asumir las exigencias institucionales y del medio, lo que da como resultado la divulgación de mayor información. La teoría institucional, la teoría de las partes interesadas y la de dependencia de recursos sostienen esta proposición.

H1: Las características de las universidades latinoamericanas como la propiedad, tamaño, capacidad de investigar y trayectoria afectarían los niveles de divulgación de información de las universidades latinoamericanas.

\section{Partes interesadas apropiativas}

La naturaleza de las relaciones entre la empresa y las partes interesadas es un tema vital. Los intereses a menudo son divergentes con la empresa o entre los grupos de interés. Donaldson y Preston (1995) aportan una taxonomía que tipifica dichas relaciones en normativas, instrumentales y descriptivas. Estas últimas abordan la manera en que la empresa debe relacionarse con sus grupos de interés; las instrumentales se preocupan de lo que pasa si la empresa se relaciona con sus grupos de interés de cierta forma; las descriptivas especifican la manera en que la empresa se relaciona con sus grupos de interés (Jones et al., 2007; Schnackenberg y Tomlinson, 2016).

Existen stakeholders de las universidades que no estarán dispuestos a promover la divulgación de información por considerar que es un valor que debe ser retenido y apropiado por aquellas partes interesadas que tienen una mayor pertenencia con la institución, como los académicos, estudiantes, ejecutivos, funcionarios y egresados de la institución.

De acuerdo a estos antecedentes, se propone la siguiente hipótesis: 
H2: las partes interesadas apropiativas de las universidades se relacionan negativamente con los niveles de divulgación de información de las universidades.

\section{Capacidad de generar información}

La divulgación de la información requiere de la capacidad de producirla y distribuirla de manera eficiente, máxime si la divulgación se mide con base en los contenidos generados por un adecuado y eficaz instrumento, como las páginas Web (Midin et al., 2017; Gandia et al., 2016; Nazuk y Shabbir, 2018; Da-Silva et al., 2017; Carrillo et al., 2017).

Svard (2017) indica que no es posible realizar una divulgación eficiente y eficaz si no se cuenta con una infraestructura de gestión de la información para facilitar la creación, captura, gestión y difusión, preservación y reutilización de la información, esto implica recursos y, como indican Bearfield y Bowman (2017), el tamaño de las organizaciones afecta el logro de la divulgación, ya que las más pequeñas tendrán menos recursos para desarrollar una capacidad de divulgación acorde a las necesidades y por eso, para el caso estudiados por ellos, las municipalidades sugieren que se utilicen recursos gubernamentales para la falta de recursos.

De acuerdo a estos antecedentes, se propone la siguiente hipótesis:

H3: La capacidad autónoma de generar información de las universidades se relaciona positivamente con la divulgación de la información de las universidades latinoamericanas hacia sus diversas partes interesadas.

\section{Atributos de los gobiernos corporativos}

Los atributos de los gobiernos corporativos, específicamente de los directorios, son tres: a) composición, b) estructura de liderazgo y c) compensación. Dentro de la composición se considera: a) tamaño, b) diversidad, c) independencia y d) especialidad funcional. En la estructura de liderazgo se establecen dos posibilidades: a) estructura separada entre el Rector y el presidente del MCC y b) dualidad o doble rol (presidente y rector con la misma persona). Al respecto, se observaron dos atributos: composición y liderazgo, atendiendo a que son posibles de medir en las universidades. De la composición no se considera la especialidad funcional, ya que no se cuenta con esa información.

Dado que existen diversas variables dentro de los atributos de los gobiernos corporativos, se establece la siguiente hipótesis, pero posteriormente se desprenden sub-hipótesis para distintos elementos relevantes dentro de la misma dimensión:

H4: Los atributos de los Máximos Cuerpos Colegiados (MCC) influyen sobre la divulgación de la información de las universidades latinoamericanas.

\section{Composición de los Máximos Cuerpos Colegiados (MCC)}

La composición del Máximo Cuerpo Colegiado, caracterizada principalmente por el tamaño, independencia y diversidad de los MCC, implica atributos que permiten explicar el comportamiento de los niveles de divulgación de la información de organizaciones complejas como las universidades (Mackey, 2011; Kim y Ozdemir 2014; Cunha y Rodrigues, 2018; Said at al., 2018). 


\section{Tamaño del MCC}

El tamaño corresponde al número de miembros que forman parte del MCC y tiene incidencia en el rol del directorio (John et al., 2016), como el ejercer el monitoreo o el aprovisionamiento de recursos. En el plano de las teorías, Abello y Mancilla (2018) indican que la teoría institucional y la teoría de la dependencia de los recursos apoyan la idea de que a mayor tamaño existirá una mayor divulgación de información en las universidades. Por ello, se propone la siguiente hipótesis:

H4.1: El tamaño del Máximo Cuerpo Colegiado se relaciona positivamente con la divulgación de información de las universidades latinoamericanas hacia los distintos grupos de interés.

\section{Independencia de los miembros del MCC}

Existen razones para indicar que un mayor porcentaje de miembros independientes de un directorio se asocia a la cantidad de información divulgada a los distintos grupos de interés (Basuony, Mohamed, y Samaha, 2018; Abello y Mancilla, 2018; Said et al., 2018). Se espera que existan incentivos para promover una mayor transparencia y con ello una mayor divulgación de información.

H4.2: la independencia de los miembros del Máximo Cuerpo Colegiado se relaciona positivamente con los niveles de divulgación de la información de las universidades latinoamericanas hacia sus diversos grupos de interés.

De manera complementaria, considerando la preponderancia de MCC endogámicos, es decir aquellos conformados solo por miembros internos, se formula la siguiente hipótesis:

H4.3: la existencia únicamente de miembros internos en el Máximo Cuerpo Colegiado se relaciona negativamente con los niveles de divulgación de la información de las universidades latinoamericanas dirigida a sus diversos grupos de interés.

\section{Diversidad de los Máximos Cuerpos Colegiados MCC}

Como indican Abello y Mancilla (2018), existen diferentes formas de representar la diversidad, ya sea si se analiza desde la diferencia de opinión (separación), de tipos o categorías (variedad) o de concentración de recursos y/o estatus. En el caso de los MCC que están conformado por distintos grupos de interés, podemos encontrar las tres tipos de diferencias.

En esta investigación se testean dos, las que tienen relación con los tipos o categorías de los grupos o partes interesadas y la concentración de miembros dentro de los grupos de interés. Esto último es importante de verificar, ya que el MCC puede estar conformado por diversos grupos, pero un grupo puede aglutinar gran parte de los miembros, lo que hace difícil evidenciar las diferencias.

La relación entre la diversidad y la divulgación de información es apoyada, ya que amplía el rango cognitivo y el comportamiento de los consejos, mejorando la calidad de los debates e incrementando la capacidad de supervisión (Ferrero-Ferrero et al., 2015; Briano-Turrent y Saavedra-García, 2015).

H4.4: la diversidad de los grupos de interés que conforman el Máximo Cuerpo Colegiado se relaciona positivamente con la divulgación de la información de las universidades latinoamericanas.

\section{Estructura de liderazgo del Máximo Cuerpo Colegiado (MCC)}

La literatura muestra que la dualidad acrecienta la retención de información y disminuye la calidad en la divulgación voluntaria de la información corporativa (Acar y Ozkan, 2017; Alfraih y Almutawa, 2017). Si bien los resultados empíricos no son concluyentes (Jizi et al., 2014; Torchia y Calabro, 2016; y Giannarakis et al., 2014), tiene más solidez 


\section{J. B. Abello Romero, et al. / Contaduría y Administración, 64(4) Especial Gobierno Corporativo, 2019, 1-17}

http://dx.doi.org/10.22201/fca.24488410e.2019.2449

teórica la relación negativa entre la dualidad del presidente del directorio y el máximo ejecutivo (gerente) y la divulgación voluntaria de la información (De Maere et al., 2014), sin haber claridad sobre quién representa la causa o el efecto. Por otra parte, la falta de un mercado de control y la dualidad puede generar una compleja situación para la firma (John et al., 2016). Donde sí se encontró plena coincidencia, es en que la dualidad podría restringir aún más la difusión de información hacia los otros miembros del directorio y a los distintos grupos interesados (Haan y Vlahu, 2016). Considerando los antecedentes expuestos, se plantea la siguiente hipótesis:

H4.5: la dualidad en la estructura de liderazgo del Máximo Cuerpo Colegiado se relaciona negativamente con los niveles de divulgación de información de las universidades latinoamericanas.

\section{Metodología}

En esta sección se presenta la metodología utilizada en este trabajo, detallándose los antecedentes recopilados, el tratamiento de los datos y análisis aplicados a los mismos.

Para llevar adelante este estudio, se consideraron 219 universidades latinoamericanas presentes en el Scimago Institutions Rankings (SIR) de Iberoamérica que están en el grupo de los primeros 500 lugares. Se seleccionaron las universidades cuya aparición en el ranking fue continua entre los años 2012 y 2015 y que se distribuyen en 5 países: Argentina (31 universidades), Brasil (98 universidades), Chile (28 universidades), Colombia (28 universidades) y México (34 universidades). Los países estudiados representan el 83\% de las universidades latinoamericanas y, en materia de publicaciones científicas, el 89\% del total de la región.

El modelo que se estudia está basado en las teorías señaladas anteriormente y se estimará por medio de mínimos cuadrados ordinarios (MCO). Este modelo consiste en que el índice de divulgación de información (IDI) está en función de cuatro dimensiones que poseen a su vez diferentes variables explicativas: las características de las universidades, partes interesadas (los stakeholders apropiativos), la capacidad de generar información y los atributos de los máximos cuerpos colegiados (MCC), más un término de perturbación estocástico (ecuación 1). No obstante, dentro de cada una de las cuatro dimensiones que se trabajan existe una serie de variables que permiten operacionalizar las dimensiones y conceptos que hay detrás de ellas.

En la ecuación [2] se puede observar el modelo completo con las variables a utilizar en este trabajo. Nótese en esta expresión que el coeficiente estimado posee un subíndice con 2 números: el primero de ellos identifica la dimensión que incide sobre la divulgación de información y el segundo término numera la variable que está siendo utilizada en este trabajo.

En la tabla 1 se entrega el detalle de las variables explicativas junto con los valores medios y desviación estándar. Al modelo se le aplican los test de normalidad de errores, test del modelo global prueba F, pruebas individuales para los coeficientes (prueba t, prueba de muticolinealidad, test de inflación de varianza) y de heterocedasticidad.

$$
\mathrm{IDI}_{\mathrm{i}}=\widehat{\beta}_{0}+\widehat{\beta}_{1} \text { Características Universidades } \mathrm{i}_{\mathrm{i}}+\widehat{\beta}_{2} \text { Partes Interesadas }_{\mathrm{i}}+\widehat{\beta}_{3} \text { Capacidad de generar información }_{\mathrm{i}}+\widehat{\beta}_{4} \text { Atributos }_{\mathrm{i}}+\varepsilon_{\mathrm{i}}
$$

IDI $_{i}=\hat{\beta}_{0}+\hat{\beta}_{11}$ Propiedad Universidades ${ }_{i}+\hat{\beta}_{12}$ Tamaño Universidades $_{i}+\hat{\beta}_{13}$ Capacidad para Investigar $_{i}+\hat{\beta}_{14}$ Trayectoria $_{i}+$ $\hat{\beta}_{21}$ Funcionarios $_{i}+\hat{\beta}_{22}$ Egresados $_{i}+\widehat{\beta}_{31}$ Capacidad para generar información $_{i}+\widehat{\beta}_{41}$ Tamaño $_{\text {MCC }}+\widehat{\beta}_{42}$ Independencia $_{i}+$ $\widehat{\beta}_{43}$ Endogámico $_{i}+\widehat{\beta}_{54}$ Diversidad de los MCC en función de los grupos de interés $s_{i}+\widehat{\beta}_{45}$ Diversidad de los grupos de interés $_{i}+\widehat{\beta}_{46}$ Dualidad de la estructura de liderazgo del $\mathrm{MCC}_{\mathrm{i}}+\varepsilon_{\mathrm{i}}$ 
Tabla 1

Descripción de las variables explicativas del modelo

\begin{tabular}{|c|c|c|c|c|}
\hline \multicolumn{2}{|c|}{$\begin{array}{l}\text { Dimensiones/Variables } \\
\text { Independientes }\end{array}$} & \multirow[t]{2}{*}{ Descripción } & \multirow{2}{*}{ Media } & \multirow{2}{*}{$\begin{array}{c}\begin{array}{c}\text { Desviación } \\
\text { estándar }\end{array} \\
0,40\end{array}$} \\
\hline \multirow{4}{*}{ 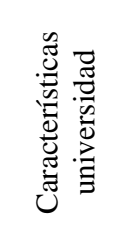 } & Propiedad & & & \\
\hline & $\begin{array}{l}\text { Tamaño } \\
\text { Universidades }\end{array}$ & $\begin{array}{l}\text { Se considera la cantidad de estudiantes normalizándola por } \\
\text { país. }\end{array}$ & 0,16 & 0,19 \\
\hline & $\begin{array}{l}\text { Capacidad de } \\
\text { investigar }\end{array}$ & Académicos con grado de doctor/total de académicos. & 0,29 & 0,25 \\
\hline & Trayectoria & Años de antigüedad de la universidad. & 59,43 & 48,58 \\
\hline \multirow{2}{*}{ 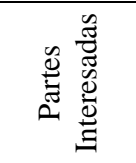 } & Funcionarios & $\begin{array}{l}\text { Corresponde a la cantidad de funcionarios que son miembros } \\
\text { con derecho a voz y voto en el Máximo Cuerpo Colegiado. }\end{array}$ & 2,55 & 3,17 \\
\hline & Egresados & $\begin{array}{l}\text { Corresponde a la cantidad de egresados que son miembros con } \\
\text { derecho a voz y voto en el Máximo Cuerpo Colegiado. }\end{array}$ & 0,51 & 1,27 \\
\hline 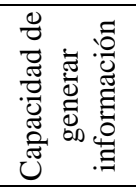 & $\begin{array}{l}\text { Capacidad } \\
\text { dominio } \\
\text { principal }\end{array}$ & $\begin{array}{l}\text { Posición Ranking webometrics de la variable presencia que } \\
\text { representa el número total de páginas web alojadas en el } \\
\text { dominio web principal (incluyendo todos los subdominios y } \\
\text { directorios) de la universidad, obtenido del mayor motor de } \\
\text { búsqueda comercial (Google). }\end{array}$ & 22.943 & 2.969 \\
\hline \multirow{6}{*}{ 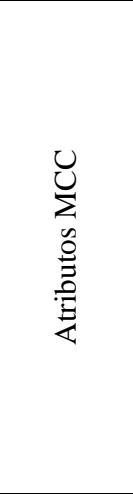 } & Tamaño MCC & Número de miembros con derecho a voto. & 44,84 & 49,32 \\
\hline & $\begin{array}{l}\text { Independencia } \\
\text { MCC }\end{array}$ & $\begin{array}{l}\text { Porcentaje de miembros externos al total de miembros del } \\
\text { MCC. }\end{array}$ & 0,19 & 0,26 \\
\hline & Endogámico & $\begin{array}{l}\text { Es una variable dicotómica donde los MCC que solo tienen } \\
\text { miembros internos }=1 \text { y MCC con miembros externos }=0\end{array}$ & 0,22 & 0,42 \\
\hline & $\begin{array}{l}\text { Diversidad del } \\
\text { MCC }\end{array}$ & $\begin{array}{l}\text { Cantidad de tipos de grupos de interés que forman parte del } \\
\text { MCC dividido por la cantidad teórica de grupos de interés (11 } \\
\text { grupos). }\end{array}$ & 0,43 & 0,14 \\
\hline & $\begin{array}{l}\text { Diversidad de los } \\
\text { grupos de interés }\end{array}$ & $\begin{array}{l}\text { Corresponde a la diversidad respecto de los grupos de interés } \\
\text { y se determina (1-HHN). HHN es el índice de Hirschman } \\
\text { Herfindhal Normalizado (Lis-Gutiérrez, 2013). }\end{array}$ & 0,824 & 0,16 \\
\hline & Dualidad & $\begin{array}{l}\text { Es una variable dicotómica donde: rectores con doble rol }=1 \\
\text { y universidades con separación de roles }=0\end{array}$ & 0,81 & 0,39 \\
\hline \multicolumn{3}{|c|}{ Número de observaciones } & \multicolumn{2}{|c|}{219} \\
\hline
\end{tabular}

Fuente: elaboración de los autores.

Las variables Diversidad del MCC y Diversidad de los grupos de interés, a pesar de que usan una misma información para realizar el cálculo correspondiente, proporcionan información diferente. De un lado, Diversidad del MCC solo identifica la proporción de los distintos grupos de interés (de todos los potenciales) que existen dentro del gobierno corporativo, esto es independiente del peso relativo que puedan tener dentro de este. Por su parte, Diversidad de los grupos de interés, tal como se explica en la tabla 1, es un indicador que se obtiene haciendo el cálculo de 1 - índice de Hirschman Herfindhal normalizado (1-HHN). Así, el indicador igual cercano a 0 indicaría que en los grupos existentes el peso relativo en cuanto número de miembros estaría concentrado en pocos grupos de interés (incluso existiendo todos los grupos de interés posible). En cambio, valores alejados de 0 y más cercanos a 1 indicarían no solo que hay varios grupos de interés, sino que además el número de miembros de los grupos interesados está distribuido de forma más equitativa.

Para verificar que estas variables y todas las del modelo no generen un problema de transgresión de supuestos en la estimación por MCO, se calculó el factor de inflación de varianza (VIF) para cada una de ellas. Un valor del VIF superior a 10 indicaría un potencial problema de muticolinealidad. Los resultados arrojaron que ninguna variable superó dicho umbral y el valor más alto fue de 5,141. En el caso específico de diversidad de MCC, se obtuvo un VIF de 1,542 y la Diversidad de los grupos de interés fue de 1,402.

Para analizar la divulgación de la información de las universidades se utilizó el índice de divulgación de información (IDI) propuesto por Abello (2018) y utilizado en Abello et al. (2018). Este consta de cinco dimensiones que 


\section{J. B. Abello Romero, et al. / Contaduría y Administración, 64(4) Especial Gobierno Corporativo, 2019, 1-17}

\section{http://dx.doi.org/10.22201/fca.24488410e.2019.2449}

tienen la misma ponderación relativa (1/5 cada una). A su vez, cada dimensión tiene distintos componentes (en total 40). A estos componentes se le asigna valor 1 (uno) si están presentes y valor 0 (cero) si están ausentes. Los datos para calcular el índice se obtuvieron desde los sitios web de las universidades de la muestra, en un periodo de seis meses. Posteriormente, se promedia el resultado para cada dimensión y luego se realiza la suma ponderada, tal como se indica se muestra en la ecuación [3]. De esta manera, con un IDI más cercano a uno la universidad divulga más información, y cuánto más cercano sea a 0 , la divulgación será más baja.

$$
\mathrm{IDI}=\left(\sum_{\mathrm{K}=1}^{\mathrm{K}=6} \frac{\mathrm{DII}}{6}\right) * 0,2+\left(\sum_{\mathrm{K}=1}^{\mathrm{K}=8} \frac{\mathrm{DIF}}{8}\right) * 0,2\left(\sum_{\mathrm{K}=1}^{\mathrm{K}=7} \frac{\mathrm{DITL}}{7}\right) * 0,2\left(\sum_{\mathrm{K}=1}^{\mathrm{K}=5} \frac{\mathrm{DIEO}}{5}\right) * 0,2\left(\sum_{\mathrm{K}=1}^{\mathrm{K}=14} \frac{\mathrm{DICI}}{14}\right) * 0,2
$$

Donde:

$\begin{array}{ll}\text { DII } & =\text { Dimensión Información Institucional } \\ \text { DIF } & =\text { Dimensión Información Financiera } \\ \text { DITL } & =\text { Dimensión Información de Transparencia y Liderazgo } \\ \text { DIEO } & =\text { Dimensión Información Estructura y Organización } \\ \text { DICI } & =\text { Dimensión Información Capital Intelectual } \\ \mathrm{K} & =\text { número de componente }\end{array}$

Fuente: Abello (2018), Abello et al. (2018).

Para el índice, se calcula el Alfa de Cronbach para verificar la fiabilidad del IDI; al respecto, se suele indicar que un valor superior a 0,6 refleja una consistencia interna adecuada (De Castro et al., 2016; Borges y Mafra, 2014). Los resultados para el IDI calculado arrojaron un Alfa de Cronbach para un índice de ponderación igual de 0,69; y de 0,72 el basado en elementos estandarizados.

Finalmente, se debe indicar que el levantamiento de información se desarrolló con base en información secundaria. Es así como las variables relacionadas con los atributos del Máximo Cuerpo Colegiado se obtuvieron del análisis de cada uno de los estatutos de las universidades y del análisis de sus sitios web. Las variables relacionadas con el tamaño, antigüedad y tipo de propiedad se obtuvieron de las bases de datos públicas de cada uno de los cincos países establecido en la muestra. Para el caso de los datos para la construcción del índice de divulgación de información, estos fueron recogidos por medio de un trabajo de recopilación de seis meses desde los sitios web de las universidades seleccionadas. 


\section{Resultados}

En la tabla 2 se presentan los resultados obtenidos de la estimación por MCO del modelo que explica los niveles de divulgación de las universidades. En primer lugar, se observa que el test global de significancia (prueba F) es altamente significativo y que, a excepción de la constante, las dimensiones y las variables consideradas para explicar los niveles de divulgación de la información son todas significativas.

Tabla 2

Resultados estimación modelo

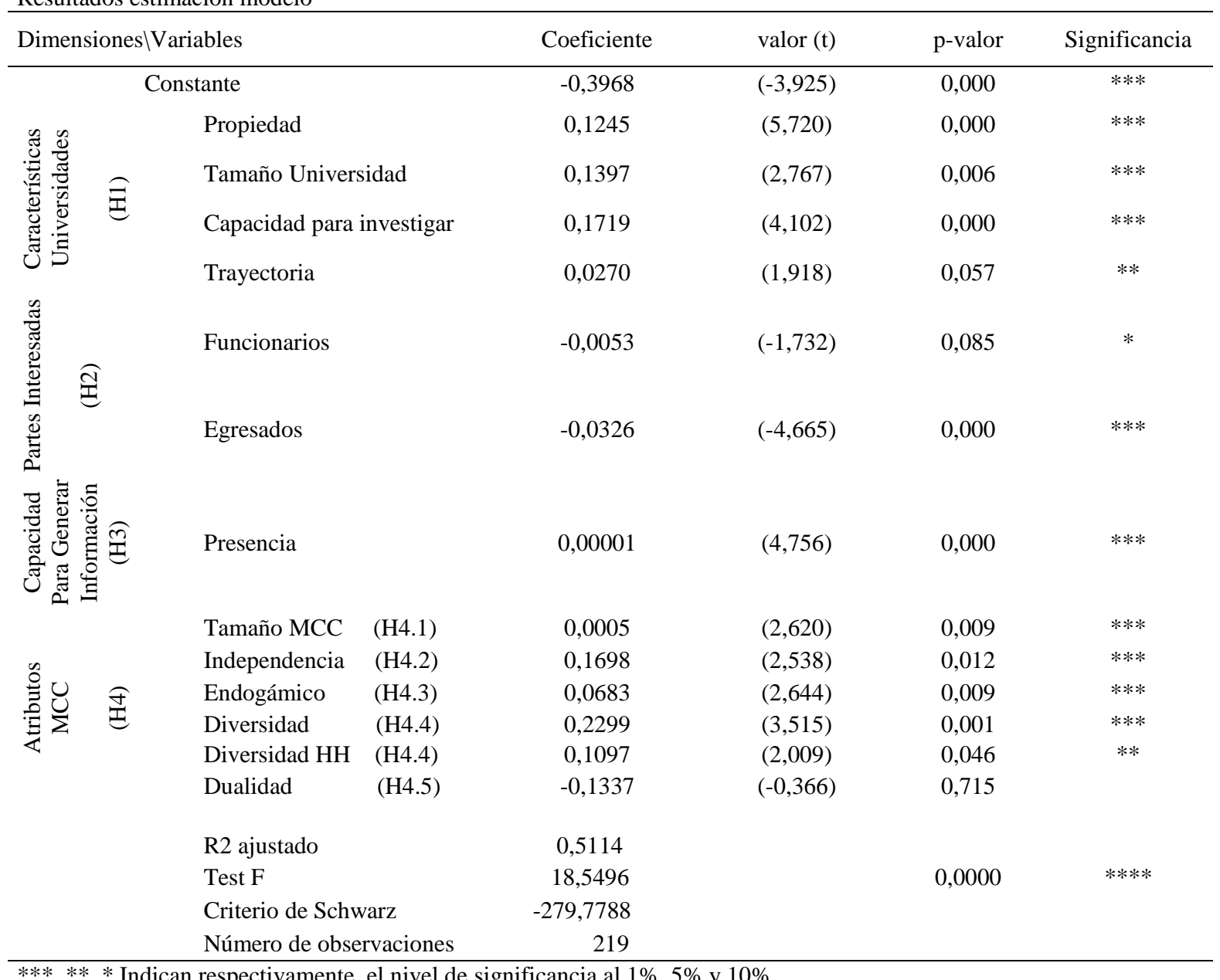

can respectivamente, el nivel de significancia al 1\%,5\% y $10 \%$

Fuente: elaboración propia.

Estos resultados, en primer lugar, permiten validar el modelo teórico propuesto en este trabajo, esquematizado en la figura 1, ya que las cuatro dimensiones que se proponen con base en las distintas teorías permiten ayudar a explicar la divulgación de información. El valor de F 18,54 con un $\mathrm{p}=0,000$ nos indica que $\mathrm{F}$ es significativa en un $\alpha=0,001$. Se rechaza la hipótesis nula, ya que existe al menos un pronosticador significativo de los niveles de divulgación de la información. Además, se acepta la hipótesis nula sobre la existencia de heterocedasticidad (con $\mathrm{p}$ valor $=\mathrm{P}($ chi-cuadrado 


\section{J. B. Abello Romero, et al. / Contaduría y Administración, 64(4) Especial Gobierno Corporativo, 2019, 1-17}

http://dx.doi.org/10.22201/fca.24488410e.2019.2449

$(99)>108,088)=0,250102$. También se realizó el contraste de normalidad de los residuos, aceptándose la hipótesis nula con Chi-cuadrado $(2)=2,04165$ con un $\mathrm{p}$ valor $=0,360298$.

$\mathrm{Al}$ revisar los resultados (tabla 2) por dimensión, se constata que todas las dimensiones aportan en la explicación de la varianza del IDI. La dimensión que refleja las características de las universidades latinoamericanas es coherente con lo planteado en el plano teórico y reafirma lo indicado por Abello et al. 2018. Todas las variables propuestas confirman la hipótesis H1. Las variables propiedad, tamaño, capacidad de generar investigación y trayectoria tienen una alta significancia y sus signos van en línea con lo propuesto. La dimensión que refleja las partes interesadas apropiativas confirma la existencia de grupos de interés que no estarán dispuestos a promover la divulgación de información, asumiendo que la retención le genera un valor a las partes interesadas de mayor pertenencia con la institución. Los funcionarios y egresados resultaron significativos y con el signo proyectado.

La capacidad para generar información de las universidades resulta ser una dimensión significativa. Se testearon diversas proxies y los resultados muestran que la variable que refleja el número total de páginas web alojadas en el dominio web principal (incluyendo todos los subdominios y directorios) de la universidad, a lo que se denomina presencia. Los atributos de los Máximos Cuerpos Colegiados (MCC) constituyen una dimensión que aporta a la explicación del comportamiento de la divulgación de información de las universidades latinoamericanas, tal como lo indican los resultados de este estudio.

Se debe resaltar el comportamiento de la variable tamaño MCC $(\mathrm{H} 4.1)$ y la variable independencia $(\mathrm{H} 4.2)$, ambas significativas a un nivel de un p<0,0. La H4.3 da significativa, pero el signo es contradictorio y se aleja de lo propuesto, lo que puede estar dado por diversas razones. Una, basada en la teoría de servidores, sostiene que a pesar de que todos los miembros del MCC sean internos, ello no inhabilita la divulgación de la información, ya que estos siempre van a inclinarse más por la institución y, junto al equipo ejecutivo, incentivarán la divulgación siempre que fuese bueno para la institución. La universidad es más importante que los intereses personales.

Por otro lado, el equipo rectoral puede tener una mejor sintonía con aquellos MCC que solo cuentan con miembros internos con poder en la organización, y conservar el poder estimulará la divulgación de la información. La variable diversidad se cuantificó de dos formas: a) de acuerdo al índice de Hirschman y Herfindhal normalizado; y b) de acuerdo a la cantidad de tipos de grupos de interés que tiene el MCC dividido por la cantidad total de grupos de interés posibles. En esta operacionalización las variables resultaron complementarias.

Con respecto al carácter dual de la estructura del liderazgo de los MCC (H 4.5), las fuentes consultadas admiten que la dualidad tiene una relación negativa con los niveles de divulgación de la información de las universidades. Los resultados de este estudio, si bien reafirman el signo de la proposición, no la relevan como significativa. Producto de la poca variabilidad que muestran los datos para el caso latinoamericano, en general los MCC tienen un liderazgo dual.

\section{Conclusiones}

Los hallazgos de este trabajo permiten sostener que este genera contribuciones en el plano teórico, empírico, metodológico y algunas implicaciones de política pública.

Desde el punto de vista teórico, el modelo que se construyó es validado por los resultados obtenidos, lo que permite sostener que las teorías en las que se basa este trabajo contribuyen a la comprensión del problema; es decir, es necesario un enfoque multi-teórico para explicar los niveles de divulgación de información de universidades. Asimismo, el modelo propuesto establece cuatro dimensiones que permiten comprender la divulgación de información, que son los 


\section{J. B. Abello Romero, et al. / Contaduría y Administración, 64(4) Especial Gobierno Corporativo, 2019, 1-17}

http://dx.doi.org/10.22201/fca.24488410e.2019.2449

atributos de los gobiernos corporativos, las características de las universidades, los grupos de interés apreciativos y la capacidad de generar información.

Desde el punto de vista empírico, para cada una de las dimensiones de modelo teórico se definieron e identificaron variables que permitieron operacionalizar la medición de tales dimensiones. Estas, junto con presentar significancia estadística, exhibieron impactos esperados según las teorías, lo que permitió validar el modelo. Mención especial se merece la variable dualidad, dentro de los atributos de los gobiernos corporativos, siendo la única variable que no presentó significancia estadística, por lo que no tendría incidencia sobre la divulgación de información por parte de las universidades.

La metodología adoptada destaca por el elevado número de observaciones, 219 universidades, y de diferentes países, junto con el hecho de recordar que estas universidades pertenecen al Scimago Institutions Rankings (SIR) de Iberoamérica y que están dentro del grupo de los primeros 500 lugares.

Desde el punto de las implicancias para políticas públicas, estos resultados proponen que se deben considerar al menos las cuatro dimensiones para incrementar los niveles de divulgación y, por lo tanto, la disminución de las asimetrías de información. Por otra parte, medidas más concretas pueden estar enfocadas, solo a modo de ejemplo, y dentro las características de las universidades, en el tamaño de las mismas. Donde los resultados indicaban que a mayor tamaño, la divulgación también es mayor, las políticas públicas deberían facilitar, incentivar o apoyar a universidades de menor tamaño.

Mientras que por el lado de los atributos de los MCC, las políticas públicas deberían tender a regular o incentivar, por ejemplo, un acrecentamiento de la diversidad e independencia en los MCC por medio de cambios en la composición (aumentando los consejeros independientes y disminuyendo los vinculados al ejecutivo), como también mediante la incorporación de otros grupos de interés.

Futuras investigaciones debieran considerar aspectos tales como un seguimiento longitudinal de los niveles de divulgación de estas universidades, así como también abrir la discusión en cuanto a la calidad y el valor de la información divulgada (y la medición de la misma).

\section{Referencias}

Abello, J. B. (2018). Impactos de los atributos de los gobiernos corporativos universitarios sobre la divulgación de información: el caso de las universidades latinoamericanas (Tesis doctoral inédita). Universidad de Santiago de Chile, Chile.

Abello, J., y Mancilla, C. (2018). Análisis Multi-Teórico de los gobiernos corporativos universitarios sobre la divulgación de información. Revista Opción, 34(86), 358-392.

Abello, J., Mancilla, C., Molina, C., y Palma, A. (2018). Relación entre divulgación de información y características universidades latinoamericanas. Revista Venezolana de Gerencia, 23(1), 67-89.

Acar, E., y Ozkan, S. (2017). Corporate governance and provisions Under IAS37. Euromed Journal of Business, 12(1), 52-72. https://dx.doi.org/10.1108/EMJ B-03-2016-0007

Alfraih, M., y Almutawa, A. (2017). Voluntary disclosure and corporate governance: empirical evidence from Kuwait. International Journal of Law and Management, 59(2), 217-236. https://dx.doi.org/10.1108/IJLMA-10-20150052

Alnabsha, A., Abdou, H., Ntim, C., y Elamer, A. (2018). Corporate boards, ownership structures and corporate disclosures: Evidence from a developing country. Journal of Applied Accounting Research, 19(1), 20-41. https://doi.org/10.1108/JAAR-01-2016-0001

Alves, H., Canadas, N., y Rodrigues, A. (2015). Voluntary disclosure, information asymmetry and the perception of governance quality: An analysis using a structural equation model. TEKHNE- Review of Applied Management Studies, 13, 66-79. http://dx.doi.org/10.1016/j.tekhne.2015.10.001

Andersen, J. (2015). How organization theory supports corporate governance scholarship. Corporate Governance, 15(4), 530-545. https://doi.org/ 10.1108/CG-02-2014-0016 


\section{J. B. Abello Romero, et al. / Contaduría y Administración, 64(4) Especial Gobierno Corporativo, 2019, 1-17}

http://dx.doi.org/10.22201/fca.24488410e.2019.2449

Arechavala-Vargas, R., y Sanchez-Cervantes, C. (2017). Las universidades públicas mexicanas: Los retos de las transformaciones institucionales hacia la investigación y la transferencia de conocimiento. Revista de Educación Superior, 46(184), 21-37. https://doi.org/10.1016/j.resu.2017.09.001

Basuony, M., Mohamed, E., y Samaha, K. (2018). Board structure and corporate disclosure via social media: An empirical study in the UK. Online Information Review, 42(5), 595-614. https://doi.org/10.1108/OIR-01-2017-0013

Bearfield, D., y Bowman, A. (2017). Can you find it on the Web? an assessment of municipal E-goverment transparency. American Review of Public Administration, 47(2), 172-188. https://doi.org/10.1177/0275074015627694

Borges, G., y Mafra, F. (2014). Ensino de contabilidade na graduacao em administracao: Uma análise sob anperspective discente. Revista de Contabilidade e Organizacöes, 8(21), 58-70. doi:https://doi.org/10.11606/rco.v8i21.55607

Briano-Turrent, G., y Saavedra-Garcia, M. (2015). La composición del consejo de administración y la escritura accionaria como factores explicativos de la transparencia en el gobierno corporativo en Latinoamerica: evidencia en empresas cotizadas de Argentina, Brasil, Chle y México. Estudios Gerenciales, 31, 275-286. https://dx.doi.org/10.1016/j.estger.2015.02.001

Brownson, R., Proctor, E., Luke, D., Baumann, A., Staub, M., Brown, M., y Johnson, M. (2017). Building Capacity for dissemination and implementation research: One university's experience. Impementation Science, 12(104), 112. https://doi.org/10.1186/s13012-017-0634-4

Carrillo, M.-V., Castillo, A., y Parejo, M. (2017). University information management through the press rooms in their websites: proposal of a management model. Informacao y Sociedade-Estudos, 27(1), 103-117. https://doi.org/10.2247/ufpb.1809-4783.2017v27n1.31168

Cormier, D., Ledoux, M., Magnan, M., y Aerts, W. (2010). Corporate governance and information asymetry between managers and investors. Corporate Governance, 10(3), 574-589. https://doi.org/ 10.1108/14720701011085553

Cunha, V., y Rodrigues, L. (2018). Determinants of structure of corporate governance disclosure in portugal. RBGNRevista Brasileira de Gestao de Negocios, 20(3), 338-360. https://doi.org/10.7819/rbgn.v0i0.3359

Da-Silva, F., Correia, M., Martins, T., y Silva, O. (2017). Analysis of disclosures on the internet of public companies in Latin America. Revista de Gestao Financas e Contabidade, 7(3), 4-22.

Davis, J., Schoorman, D., y Donaldson, L. (1997). Toward a stewardship theory of management. Academy of Manegement, 22(1), 20-47. https://doi.org/10.2307/259223

De Castro, M., Jacometti, M., y Goncalves, S. (2016). Governance in the context of inter-organizational networks: A study in local productive arrangements from Parana state regarding the institutional approach. Revista Electrónica de Ciencia Administrativa, 15(2), 90-115. https://doi.org/10.21529/RECADM.2016008

De Maere, J., Jorissen, A., y Uhlaner, L. (2014). Board Capital and the downward spiral: antecedents of bankruptcy in a sample of unlisted firms. Corporate governance: An International Review, 22(5), 387-407. https://doi.org/10.1111/corg.12078

Deb, P., y Wiklund, J. (2017). The effects of CEO founder status and stock ownership on entrepreneurial orientation in small firms. Journal of Small Business Management, 55(1), 32-55. https://doi.org/10.1111/jsbm.12231

Dimaggio, P., y Powell, W. (1983). The iron cage revisited: Institutional isomorphism and collective rationality in organizational fields. American Sociological Review, 48(2), 147-160. https://doi.org/10.2307/2095101

Dolinsek, T., y Lutar-Skerbinjek, A. (2018). Voluntary disclosure of financial information on the internet by large companies in slovenia. Kybernetes, 47(3), 458-473. https://doi.org/10.1108/K-08-2016-0220

Donaldson, T., y Preston, L. (1995). The stakeholder theory of the corporation: concepts, evidence, and complications. The Academy of Management Review, 20(1), 65-91. https://doi.org/10.5465/amr.1995.9503271992

Elshandidy, T., y Shrives, P. (2016). Environmental Incentives for and usefulness of textual risk reporting: Evidence from Germany. The International Journal of Accounting, 51(4), 464-486. https://doi.org/10.1016/jintacc.2016.10001

Fay, D., y Zavattaro, S. (2016). Branding and isomorphism: The case of higther education. Public Administration Review, 76(5), 805-815. https://doi.org/10.1111/puar.12626

Felix, L., Costa-Souza, G., y Gois de Oliveira, M. (2016). Comparative analysis of factors that affects the disclosure of information related to human resources (2004-2013). Revista de Gestao Financas e Contabilidade, 6(3), 62-75. https://doi.org10.18028/2238-5320/rgfc.v6n3p62-75

Ferrero-Ferrero, I., Fernández-Izquierdo, M., y Muñoz-Torres, M. (2015). Integrating sustainability into corporate governance: An empirical study on board diversity. Corporate Social Responsability and Environmental Management, 22, 193-207. https://doi.org/10.1002/csr.1333

Ferrero-Ferrero, I., Fernandez-Izquierdo, M., Muñoz-Torres, M., y Belles-Colomer, L. (2018). Stakeholder engagement in sustainability reporting in higher education an analysis of key internal stakeholders' expectations. International Journal of Sustainability in Higher Education., 19(2), 313-336. https://doi.org/10.1108/IJSHE-06-2016-0116

Fowles, J., Frederickson, G., y Kopell, J. (2016). University Rankings: Evidence and a conceptual framework. Public Administration Review, 76(5), 790-803. https://doi.org/10.1111/puar.12610

Freeman, E. (1984). Strategic Management: A Stakeholder Approach. Boston: Pitman.

Freeman, E., Wicks, A., y Parmar, B. (2004). Stakeholder theory and "the corporate objective revisited". Organization Science, 15(3), 364-369. https://doi.org/10.1287/orsc.1040.0066

Frost, J., y Hattke, F. (2018). Governing collective action- the impetus for university commons. European Review, 26, 7084. https://doi.org/10.1017/S1062798717000552 


\section{J. B. Abello Romero, et al. / Contaduría y Administración, 64(4) Especial Gobierno Corporativo, 2019, 1-17}

http://dx.doi.org/10.22201/fca.24488410e.2019.2449

Gandía, J., Marrahi, L., y Huguet, D. (2016). Digital transparency and Web 2.0 in spanish city councils. Goverment Information Quarterly, 33(1), 28-39. https://doi.org/10.1016/j.giq.2015.12.004

Garas, S., y Elmassah, S. (2018). Corporate governance and corporate social responsability disclosure: The case of GCC countries. Critical Perspectives on International Business, 14(1), 2-26. https://doi.org/10.1108/cpoib-10-20160042

García-Sánchez, I., y Noguera-Gámez, L. (2017). Integrated reporting and stakeholder engagement: The effect on information asymmetry. Corporate Social Responsibility and Environmental Management., 24(5), 395-413. https://doi.org/10.1002/csr.1415

Garde-Sánchez, R., Rodriguez, M., y López, A. (2017). Corporate and managerial characteristics as drivers of social responsability disclosure by state-owned enterprises. Review Managerial Science, 11(3), 633-659. https://doi.org/10.1007/s11846-016-0199-7

Garrity, B. (2015). Trustees Versus Directors, Whom Do They Serve? Boards, For-Profits and the Public Good in the United States. Higher Education Quarterly, 69(1), 37-57. https://doi.org/10.1111/hequ.12057

Gianiodis, P., y Markman, G. (2016). Entrepreneurial universities and overt opportunism. Small Business Economics, 47(3), 609-631. https://doi.org/10.1007/s11187-016-9753-6

Giannarakis, G., Konteos, G., y Sariannidis, N. (2014). Financial, governance and environmental determinants of corporate social responsible disclosure. Management Decision, 52(10), 1928-1951. https://doi.org/10.1108/MD05-2014-0296

Haan, J., y Vlahu, R. (2016). Corporate Governance of Banks a survey. Journal Economic Surveys, 30(2), $228-277$. https://doi.org/10.1111/joes.12101

Hambrick, D., y Mason, P. (1984). Upper echelons: The organization as a reflection of its top managers. Academy of Management Review, 9(2), 193-206. https://doi.org/10.5465/amr.1984.4277628

Harris, M. (2013). Understanding Institutional diversity in American Higher Education. (M. Harris, Ed.) J-B Ashe Higher Education Report, 39(3), 1-152. https://doi.org/10.1002/aehe.20009

Harsh, M., Bal, R., Wetmore, J., Zachary, P., y Holden, K. (2018). The rise of computing research in East Africa: The relationship between funding, capacity and research community in a nascent field. Minerva, 56(1), 35-58. https://doi.org/10.1007/s11024-017-9341-1

Hoskisson, R., Chirico, F., Zyung, J., y Gambeta, E. (2017). Managerial risk talking: A multitheoretical review and future research agenda. Journal of Management, 43(1), 137-169. https://doi.org/10.1177/0149206316671583

Huenneke, L., Stearms, D., Martinez, J., y Laurela, K. (2017). Key strategies for building research capacity of university members. Innovative Higher Education, 42(5-6), 421-435. https://doi.org/10.1007/s10755-017-9394-y

Jensen, M., y Meckling, N. (1976). Theory of the Firma: managerial behavior, agency cost and ownership structure. Journal of Financial of Economics, 3, 305-360. https://doi.org/10.2139/ssrn.94043

Jizi, M., Salama, A., Dixon, R., y Stralling, R. (2014). Corporate Governance and corporate social responsability disclosure: Evidence from the US banking sector. Journal of Business Ethics, 125(4), 601-615. https://doi.org/10.1007/s10551-013-1929-2

John, K., Demasi, S., y Paci, A. (2016). Corporate Governance in Banks. Corporate Governance: An International Review, 24(3), 303-321. https://doi.org/10.1111/corg.12161

Jones, T., Felps, W., y Bigley, G. (2007). Ethical theory and stakeholders-related decisions: The role of stakeholders culture. The Academy of Management Review, 32(1), 137-155. https://doi.org/10.5465/amr.2007.23463924

Khlif, H., Ahmed , K., y Souissi, M. (2017). Ownership structure and voluntary disclosure: A synthesis of empirical studies. Australian Journal of Management, 42(3), 376-403. https://doi.org/10.1177/0312896216641475

Kim, Y., y Ozdemir, S. (2014). Structuring Coporate Boards for wealth protection and or wealth creation: the effects of National Institutional Characteristics. Corporate Governance: An International Review, 22(3), 266-289. https://doi.org/10.1111/corg.12062

Kohoutek, J., Pinlteiro, R., Cabelkova, I., y Smidova, M. (2017). High Education institutions in peripheral regions: A literature review and framework of analysis. Hogher Education Policy, 30(4), 405-423. https://doi.org/10.1057/s41307-017-0062-8

Kotsemir, M., y Shashnov, S. (2017). Measuring, analysis and visualization of research capacity of university at the level of departaments and staff members. Scientometrics, 112(3), 1659-1689. https://doi.org/10.1007/s11192-0172450-7

Krause, R., Semadeni, M., y Withers, M. (2016). That special someone: when the boards views its chair as a resource. Strategic Management Journal, 37, 1990-2002. https://doi.org/10.1002/smj.2444

Kultys, J. (2016). Controversies about agency theory as theoretical basis for corporate governance. Quarterly Journal Oeconomia Copernica, 7(4), 613-634. https://doi.org/10.12775/OeC.2016.034

Kuo, H., Wang, L., y Yeh, L. (2018). The role of education of directors in influening firm RyD investment. Asia Pacific Management Review, 23(2), 108-120. https://doi.org/10.1016/j.apmrv.2017.05.002

Lis-Gutiérrez, J. (31 de mayo de 2013). Superintendencia industria y comercio. Obtenido de:http://www.sic.gov.co/sites/default/files/files/estudios\%20economicos\%20sectoriales/DT012\%20_v2015_.pd f y consultado: 15/01/2019

Mackey, R. (2011). University governing boards and the risk of agency capture: A study of board members' interest group affiliations. Journal of Organizational Learning and Leadership, 9(1), 10-21. 


\section{J. B. Abello Romero, et al. / Contaduría y Administración, 64(4) Especial Gobierno Corporativo, 2019, 1-17}

http://dx.doi.org/10.22201/fca.24488410e.2019.2449

Madison, K., Holt, D., Kellermanns, f., y Ranft, A. (2016). Viewing family firm behavior and governance trough the lens of agency and stewardship theories. Family Business Review, 29(1), 65-93. https://doi.org/10.1177/0894486515594292

Marginson, S. (2017). The public good created by higher education institutions in Russia. Voprosy ObrazavamyaEducational Studies Moscow, 3, 8-36. https://doi.org/10.17323/1814-9545-2017-3-8-36

Midin, M., Joseph, C., y Mohamed, N. (2017). Promoting societal governance: stakeholders disclosure on Malaysian local authorities' websites. Journal of Cleaner Production, 142(4), 1672-1683. https://doi.org/10.1016/j.jclepro.2016.11.122

Molio, T. (2016). Governance of risks in south Africa's public education institutions (HELS). Investment Management and Financial Innovations, 13(2), 226-234. http://dx.doi.org/10.21511/imfi.13 (2-1).2016.09

Mtawa, N., Fongwa, S., y Wangenge-Ouma, G. (2016). The scholarship of university-community engagement: Interrogating Boyer's model. International Journal of Educational Development, 49, 126-133. https://doi.org/10.1016/j.ijedudev.2016.01.007

Nagata, K., y Nguyen, P. (2017). Ownership structure and disclosure quality evidence from management forecats revisions in Japan. Journal Accounting and Public Policy, 36(6), 451-467. https://doi.org/10.1016/j.jaccpubpol.2017.09.003

Nazuk, A., y Shabbir, J. (2018). A new disclosure index for non-governmental organizations. Plos One, 13(2). https//doi.org/10.1371/journal.pone.0191337

Nurunnabi, M. (2015). tensions between politico-institutional factors and accounting regulation in a developing economy: insights from institutional theory. Business Ethics: A European Review, 24(4), 398-424. https://doi.org/10.1111/beer.12089

OCDE. (2016). Principios de Gobierno Corporativo de la OCDE y del G20. Paris: Editions OCDE. https://doi.org/10.1787/9789264259171-es

OEI. (2010). 2021 Metas educativas- documento final. Madrid: Organización de Estados Iberoamericanos para la Educación, la Ciencia y la Cultura. Disponible en: https://www.oei.es/Educacion/metas2021/documento-final y consultado: 10/01/2019

Perez-Calero, L., Guerrero-Villegas, J., y Hurtado, J. M. (2017). The influence of organizational factors on board roles. Management Decision, 55(5), 842-871. https://doi.org/10.1108/MD-12-2015-0566

Pfeffer, J., y Salancik, G. (1978). The external control of organizations: A resource dependence perspective. Nueva York: Harper y Row.

Pham, N. L., Oh, K. B., y Pech, R. J. (2015). Mergens and acquisitions: CEO duality, operating performance and stock returns in Vietnam. Pacific-Basin Finance Journal, 35, 298-316. https://doi.org/10.1016/j.pacfin.2015.01.007

Plöckinger, M., Aschauer, E., Hiebl, M., y Rohatscher, R. (2016). The influence individual excutives on corporate financial reporting: A review and outlook from the perspective of upper echelons theory. Journal of Accounting Literature, 37, 55-75. https://doi.org/10.1016/j.acclit.2016.09.002

Powell, J., y Dusdal, J. (2017). Science Production in Germany, France, Belgium, and Luxemburgo: Comparing the contributions of research universiries and instituties to science, technology, engineering, mathematics, and health. Minerva, 55(4), 413-434. https://doi.org/10.1007/s11024-017-9327-z

Pucheta-Martinez, M., Bel-Oms, I., y Olcina-Sempere, G. (2016). Corporate Governance, female directors and quality of financial information. Business Ethics: A European review, 25(4), 363-385. https://doi.org/10.1111/beer.12123

Rhoads, R., Zheng, M., y Sun, X. (2017). The methodological socialization of social science doctoral students in China and the USA. Higher Education, 73(2), 335-351. https://doi.org/ 10.1007/s10734-016-0023-y

Rodrigues, L., Tejedo-Romero, F., y Craig, R. (2016). Corporate governance and intellectual capital reporting in a period of financial crisis: evidence from portugal. International Journal of disclosure and governance, 14(1), 1-29. https://doi.org/10.1057/jdg.2015.20

Rodriguez, L., Gallego, I., y Garcia, I. (2010). Determinantes de la divulgación voluntaria de información estratégica en internet: Un estudio de las empresas españolas cotizadas. Revista Europea de Dirección Y Economía de la Empresa, 19(1), 9-26.

Ruiz-Jimenez, J., y Fuentes-Fuentes, M. (2016). Management capabilities innovation, and gender diversity in the top management team: An empirical analysis in technology-based SMEs. Business Research Quarterly, 19(2), 107121. https://doi.org/10.1016/j.brq.2015.08.003

Sabbaghi, O. (2016). Corporate governance in China: Review. Corporate Governance- The International Journal of Business in Society, 16(5), 866-882. https://doi.org/10.1108/CG-12-2015-0162

Said, R., Rahim, A., y Hassan, R. (2018). Exploring the effects of corporate governance and human governance on manegement commentary disclosure. Social Responsability Journal, 14(4), 843-858. https://doi.org/10.1108/SRJ-06-2017-0099

Schnackenberg, A., y Tomlinson, E. (2016). Organizational transparency anew perspective on managing trust in organization- stakeholder relationships. Journal of Management, 42(7), 1784-1810. https://doi.org/10.1177/0149206314525202

Shi, W., Pathak, S., Song, L., y Hoskisson, R. (2017). The adoption of chief diversity officers among SyP 500 firms: Institutional, resource dependence, and upper schelons accounts. Human Resour Manage, 57(1), 83-96. https://doi.org/10.1002/hrm.21837 


\section{http://dx.doi.org/10.22201/fca.24488410e.2019.2449}

Sun, J., Kent, P., Qi, B., y Wang, J. (2019). Chief financial officer demografic characteristics and fraudulent financial reporting in china. Accounting \& Finance, 59(4), 2705-2734. https://doi.org/10.1111/acfi.12286

Svard, P. (2017). Freedom of information laws and information access: The case of Sierra Leone. Information Development, 33(2), 190-198. https://doi.org/10.1177/0266666916642829

Testera, A., y Cabeza, L. (2013). Análisis de los Factores Determinates de la Transparencia en RSC en las Empresas Españolas Cotizadas. Intangible Capital, 9(1), 225-261. https://doi.org/10.3926/ic.311

Torchia, M., y Calabro, A. (2016). Board of directors and financial transparency and disclosur. Evidence from Italy. Corporate Governance-The International Journal of Business in Society, 16(3), 593-608. https://doi.org/10.1108/CG-01-2016-0019

Van Buskirk, A. (2012). Disclosure Frecuency and information asymmetry. review of Quantitative Finance and Accounting, 38(4), 411-448. https://doi.org/10.1007/s11156-011-0237-0

Vasbinder, J. (2017). What if there were no universities? Psych Journal, 6(4), 316-326. https://doi.org/10.1002/pchj.199

Wei, J., Ouyang, Z., y Chen, H. (2018). CEO characteristics and corporatephilanthropic giving in an emerging market: the case of China. Journal of Business Research, 87, 1-11. https://doi.org/10.1016/j.jbusres.2018.02.018

Yasser, Q. R., Al Mamun, A., y Rodrigs, M. (2017). Impact of board structure on firm performance: Evidence from an emerging economy. Journal of Asia Business Studies, 11(2), 210-228. https://doi.org/10.1108/JABS-06-20150067 